\title{
Computed eigenmodes of planar regions
}

Trefethen, Lloyd N. and Betcke, Timo

2005

MIMS EPrint: 2006.367

Manchester Institute for Mathematical Sciences

School of Mathematics

The University of Manchester

\footnotetext{
Reports available from: http://eprints.maths.manchester.ac.uk/

And by contacting: The MIMS Secretary

School of Mathematics

The University of Manchester

Manchester, M13 9PL, UK
} 


\title{
Computed eigenmodes of planar regions
}

\author{
Lloyd N. Trefethen and Timo Betcke
}

\begin{abstract}
Recently developed numerical methods make possible the highaccuracy computation of eigenmodes of the Laplacian for a variety of "drums" in two dimensions. A number of computed examples are presented together with a discussion of their implications concerning bound and continuum states, isospectrality, symmetry and degeneracy, eigenvalue avoidance, resonance, localization, eigenvalue optimization, perturbation of eigenvalues and eigenvectors, and other matters.
\end{abstract}

\section{Introduction}

Eigenmodes of metal plates were measured experimentally by Chladni in 1808, who became celebrated for this work, and in the generations that followed, the mathematics of eigenmodes of vibrating plates and membranes was set forth by, among others, Poisson (1829), Lamé (1861), Clebsch (1862), Weber (1869), Rayleigh (1877), Schwarz (1885), and Pockels (1891). It was soon recognized that the same mathematics describes the modes that appear in certain electromagnetic cavities. A new application came with Schrödinger's equation in 1926, which showed that these equations also govern quantum states of a particle trapped in a two-dimensional well. A further fifty years after that, these matters began to receive renewed attention with the emergence of the field of chaotic dynamics and associated questions of billiards, scars, and ergodicity. Nowadays, for every person with an interest in membranes or microwaves, there is another who speaks of "quantum chaos" [30].

The problem we shall consider is to find the eigenvalues $\lambda$ and eigenfunctions $u$ of a two-dimensional planar "drum" defined by the Helmholtz equation

$$
-\Delta u=\lambda u, \quad u=0 \text { on } \partial \Omega,
$$

where $\Omega$ is an open set in $\mathbf{R}^{2}, u$ is twice differentiable in $\Omega$ and continuous on $\bar{\Omega}$ and not identically zero, and $\Delta$ is the Laplacian $[\mathbf{2 0}, \mathbf{3 8}]$. If $\Omega$ is a bounded region with a piecewise smooth boundary, then it has a countably infinite set of eigenfunctions $81 \mathrm{Q} 50$

1991 Mathematics Subject Classification. Primary 35J05, 65N25; Secondary 35P99, 74K15,

Key words and phrases. billiards, drums, Helmholtz equation, level repulsion, membranes, quantum chaos, Schrödinger operator.

TB was supported by a Scatcherd European Scholarship. 
with eigenvalues satisfying

$$
0<\lambda_{1}<\lambda_{2} \leq \lambda_{3} \leq \ldots, \quad \lambda_{k} \rightarrow \infty .
$$

In the usual area inner product, the eigenfunctions can be taken to be orthogonal and complete, and the lowest is of just one sign. For unbounded regions with infinite area, eigenmodes may or may not exist, and in addition, there may be a continuous spectrum.

Numerical methods for solving (1.1) have also been developing with the years. We two have been involved with recent advances in this area $[\mathbf{1 3}, \mathbf{1 4}, \mathbf{1 5}]$, as have others including Bäcker, Banjai, Barnett, Descloux, Driscoll, Heller, Saraceno, Tolley, and Vergini $[\mathbf{5}, \mathbf{7}, \mathbf{8}, \mathbf{9}, \mathbf{2 2}, \mathbf{2 3}, \mathbf{3 2}, \mathbf{5 1}]$. The present paper is devoted to presenting some of the fruits of this algorithmic work. Our plan is to explore a collection of examples of planar regions with interesting spectra, and to use these examples to illustrate certain themes of mathematical and physical interest. Along the way we shall record precise numbers for possible reference by future researchers. Though we never give details of our numerical results, we report most eigenvalues to 8 digits of precision, and all digits listed are in each case believed to be correct. Only a few high-precision computed eigenvalues such as these have been reported previously in the literature.

\section{L-shaped regions-symmetries and singularities}

Of all regions $\Omega$ that cannot be treated analytically, perhaps the most familiar is the L shape, which we shall take to consist of a concatenation of three unit squares. This was the central example in the 1967 paper by Fox, Henrici and Moler that was an important early contribution to numerical methods for these problems [25], an outgrowth of Moler's $1965 \mathrm{PhD}$ thesis at Stanford. In the late 1970s, Moler took this example to illustrate the power of his new computer system MATLAB, and when MathWorks, Inc. was founded in 1984, the new company chose an image related to the first eigenmode of the $\mathrm{L}$ as its logo.

Figure 1 presents nine eigenfunctions of this region together with their eigenvalues. Here as in all our figures, each eigenfunction is scaled to have maximum value 1 , and the level curves lie at heights $-0.8,-0.6, \ldots, 0.6,0.8$, with the negative levels shown in grey. The first six of these results were computed by Fox, et al., and indeed, the first twelve eigenvalues of this region can be found hardcoded in MATLAB's membrane function.

A number of interesting features can be seen in Fig. 1. The first one we note is that as $\lambda$ increases, the characteristic wavelength of the eigenmode decreases. If we think of a typical mode as looking locally like $\sin \left(k_{x}\right) \sin \left(k_{y}\right)$ for some wave numbers $k_{x}$ and $k_{y}$ with $k^{2}=k_{x}^{2}+k_{y}^{2}$, then $k$ will satisfy $k^{2}=\lambda$; thus a typical local wave number is $k=\sqrt{\lambda}$, corresponding to a wavelength $2 \pi / \sqrt{\lambda}$. For $\lambda \approx 493$, as in our final image, this argument suggests a wavelength of about 0.28 , which is consistent with the patterns seen there. This dependence of wavelength on eigenvalue is related to Weyl's Law for eigenmodes of planar regions, which asserts that

$$
\lambda_{n} \sim \frac{4 \pi n}{A} \quad(n \rightarrow \infty)
$$

where $A$ is the area of $\Omega[\mathbf{2 0}$, sec. VI.4]. For this eigenmode we have $4 \pi n / A \approx 435.6$, which differs from the true value by about $12 \%$. (The better approximation 491.3 is obtained by adding to $(2.1)$ the term $\sqrt{4 \pi n} L A^{-3 / 2}$, where $L$ is the perimeter of 

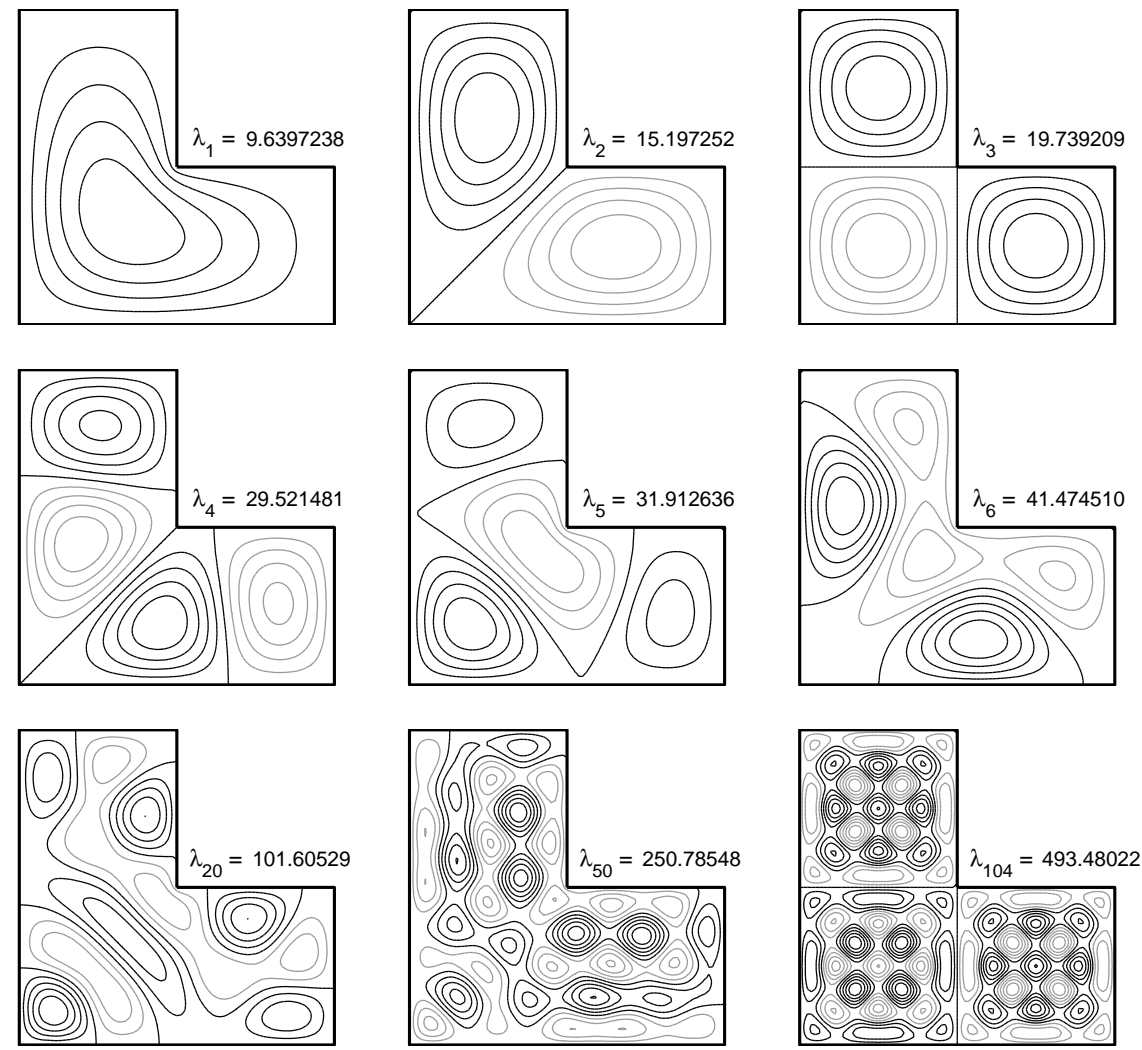

FIGURE 1. Nine eigenmodes of the L-shaped region studied by Fox, Henrici and Moler in 1967 [25]. The first eight modes shown correspond to simple eigenvalues, and are thus uniquely defined, while the last one is an arbitrary representative of a three-dimensional eigenspace associated with a degenerate triplet.

$\Omega[6]$, the starting point of the study of the Weyl expansion and other asymptotic results for (1.1) and related problems $[\mathbf{6}, \mathbf{1 0}, \mathbf{3 1}, 35]$.) There are many physical implications of these matters of the dependence of numbers of eigenmodes on volumes. For example, a comparison of volumes of $3 \mathrm{D}$ regions with the scale of Planck's constant determines how many modes can "fit in a cavity," and from this analysis can be derived the Stefan-Boltzmann formula and other laws of blackbody radiation. It was for his work in deriving these laws that Planck won the Nobel Prize in Physics in 1918.

Another notable feature of Fig. 1 is the symmetry of the region about the diagonal axis, which leads to symmetries of the eigenfunctions: each one is either even or odd with respect to the diagonal, hence satisfies either a Dirichlet or a Neumann condition there. We can explain this phenomenon as follows. Suppose a region $\Omega$ is invariant with respect to reflection across a line $L$, and let $u$ be an eigenfunction of $\Omega$ for some $\lambda$. Let $v$ be the function defined by $v(x, y)=u\left(x_{r}, y_{r}\right)$, where $\left(x_{r}, y_{r}\right)$ are the coordinates of the reflection of $(x, y)$ across $L$. Then we have $u=u_{\mathrm{e}}+u_{\mathrm{o}}$, where $u_{\mathrm{e}}=(u+v) / 2$ and $u_{\mathrm{o}}=(u-v) / 2$ are the even and odd parts 
of $u$. Clearly $u, v, u_{\mathrm{e}}$ and $u_{\mathrm{o}}$ all satisfy the eigenvalue equation for the same $\lambda$; at the same time, $u_{\mathrm{e}}$ and $u_{\mathrm{o}}$ must be distinct. If $\lambda$ is simple, we conclude that one of $u_{\mathrm{e}}$ and $u_{\mathrm{o}}$ must be zero, and thus $u$ is either odd or even with respect to $L$. If $\lambda$ is multiple, then $u$ may or may not be odd or even, but if it is neither, then $u$ and $v$ are linearly independent and $u_{e}$ and $u_{\mathrm{o}}$ are distinct eigenfunctions that span the same two-dimensional space. Thus we see that when a domain has a line of symmetry, all the functions in a basis of eigenfunctions can be taken to be symmetric or antisymmetric with respect to that line.

The L shape also has another kind of symmetry, evident in Fig. 1 in eigenmodes 3 and 104: the region can be divided into three identical squares. It follows that any eigenfunction of a single unit square (with Dirichlet boundary conditions) can be extended by two reflections to an eigenfunction of the $\mathrm{L}$ shape with the same eigenvalue. In the figure, the third eigenfunction is of this type, with $\lambda_{3}=2 \pi^{2}$, and so is the 104th, with $\lambda_{104}=50 \pi^{2}$. The latter case is particularly interesting, for this is an example of degeneracy, in fact, double degeneracy:

$$
\lambda_{103}=\lambda_{104}=\lambda_{105}=50 \pi^{2} .
$$

One can explain this by noting that 50 can be written as the sum of two squares in three different ways:

$$
50=5^{2}+5^{2}=1^{2}+7^{2}=7^{2}+1^{2},
$$

implying that $\sin (5 \pi x) \sin (5 \pi y), \sin (\pi x) \sin (7 \pi y)$, and $\sin (7 \pi x) \sin (\pi y)$ are three linearly independent eigenfunctions with this same eigenvalue. In Fig. 1 we could have chosen one of these eigenfunctions as our illustration, but it seemed more interesting to show instead the more generic choice selected arbitrarily by our numerical method.

Further discussion of symmetry of domains and eigenfunctions, and of multiplicities (which a number theory argument shows can be arbitrarily high), can be found in $[\mathbf{3 8}]$. See also Section 7, below.

Another issue that arises with the $\mathrm{L}$ shape is the singularity associated with the reentrant corner. In general, an eigenfunction (1.1) can be analytically continued outside $\partial \Omega$ near any boundary point at which $\partial \Omega$ is an analytic curve $[\mathbf{1 9}, \mathbf{2 6}, \mathbf{3 3}$, 50]. By multiple reflections, it can also be continued across any corner where $\partial \Omega$ consists locally of two straight segments meeting at an internal angle of $\pi / \alpha$, where $\alpha$ is an integer. Thus the five salient corners of the $\mathrm{L}$ shaped region are points of analyticity, with $\alpha=2$, but the reentrant corner, with $\alpha=2 / 3$, is a point of singularity. Accurate numerical methods must take such singularities into account.

Figure 2 shows another kind of L-shaped region, now with a quarter-circular curved boundary. One can interpret this region as one-quarter of a square of side length 2 with a unit circle removed - a familiar example in chaotic dynamics known as a Sinai billiard. In this case another reflection argument shows that all the corners are nonsingular, and the eigenfunctions can be analytically continued into a neighborhood of $\Omega$. Another change from the usual $\mathrm{L}$ shape is that there are no longer any degenerate eigenvalues, at least so far as we are aware.

\section{A rectangular dumbbell — broken symmetry and line splitting}

Our next example carries further the discussion of symmetry. Here $\Omega$ is a "dumbbell" consisting of two squares of side length $\pi$ coupled by a bridge of length and width $\pi / 4$. Since $\Omega$ is symmetric with respect to two axes, one sees in Fig. 3 

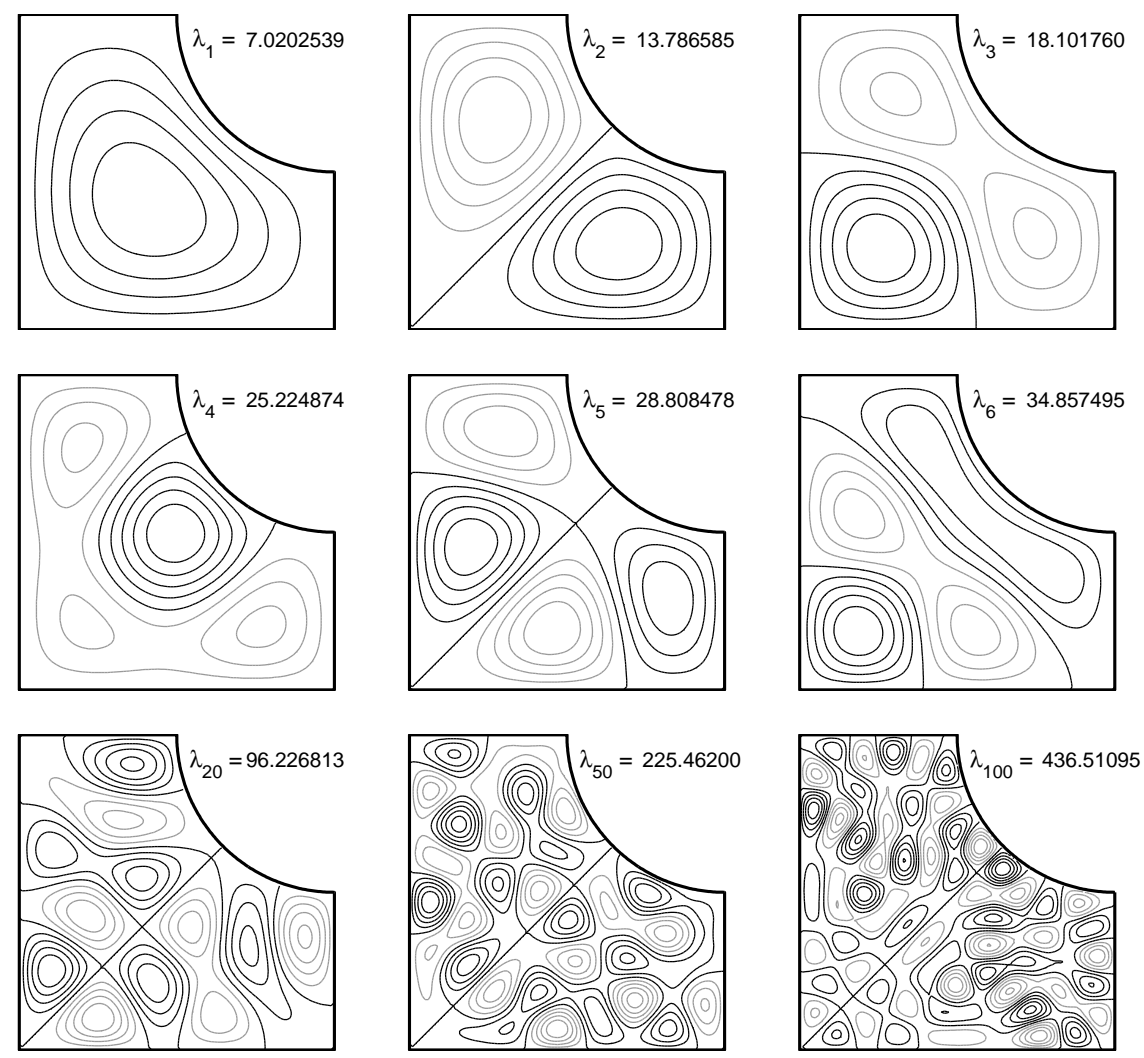

FIgURE 2. Nine eigenmodes of a curved L-shaped region (one quarter of a Sinai billiard).

that all the eigenfunctions are symmetric or antisymmetric with respect to both symmetry lines. (There are no degeneracies, so no special choices of eigenfunctions are needed to achieve these symmetries.)

The new feature of this example is that it can be viewed as two square domains that are weakly coupled. In a square of side length $\pi$, the eigenvalues are the numbers $i^{2}+j^{2}$, where $i$ and $j$ are positive integers: that is, $2,5,5,8,10,10,13,13, \ldots$ In a disconnected region consisting of two such squares uncoupled, the eigenvalues would accordingly be

$$
2,2,5,5,5,5,8,8,10,10,10,10, \ldots
$$

When the bridge is put in connecting the two squares, the eigenvalues decrease and the degeneracies are broken; for our particular choices of dimensions the first six numbers in the above sequence become approximately

$$
1.956,1.961,4.80,4.83,4.99684,4.99685 .
$$

From the figure one can get an idea of why some of the eigenvalues have shrunk farther than others. Eigenmode 1, for example, has greater amplitude in the bridge region than eigenmode 2 , which explains why $\lambda_{1}$ is further than $\lambda_{2}$ from 2 . As 

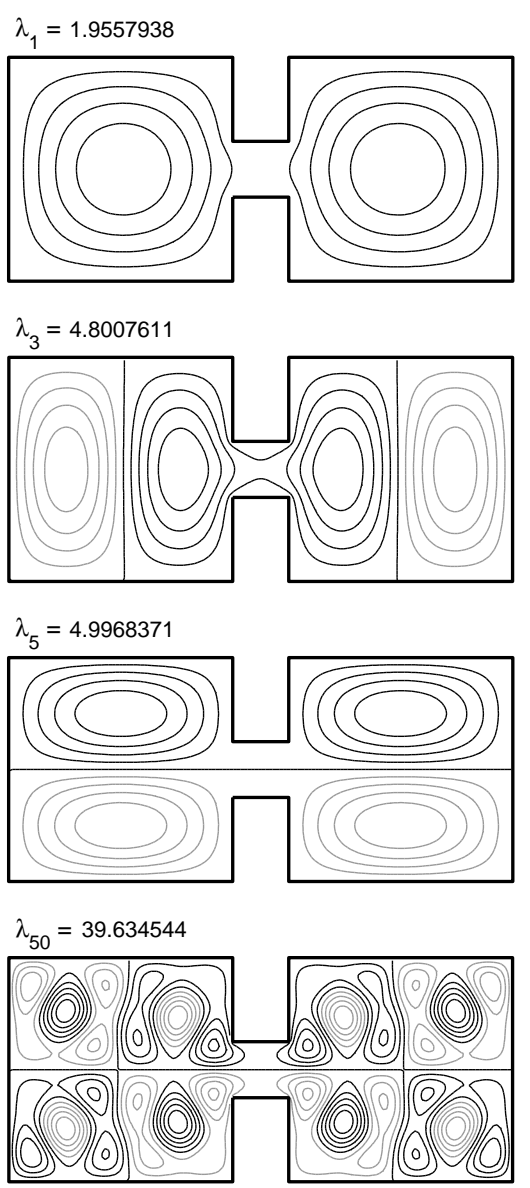
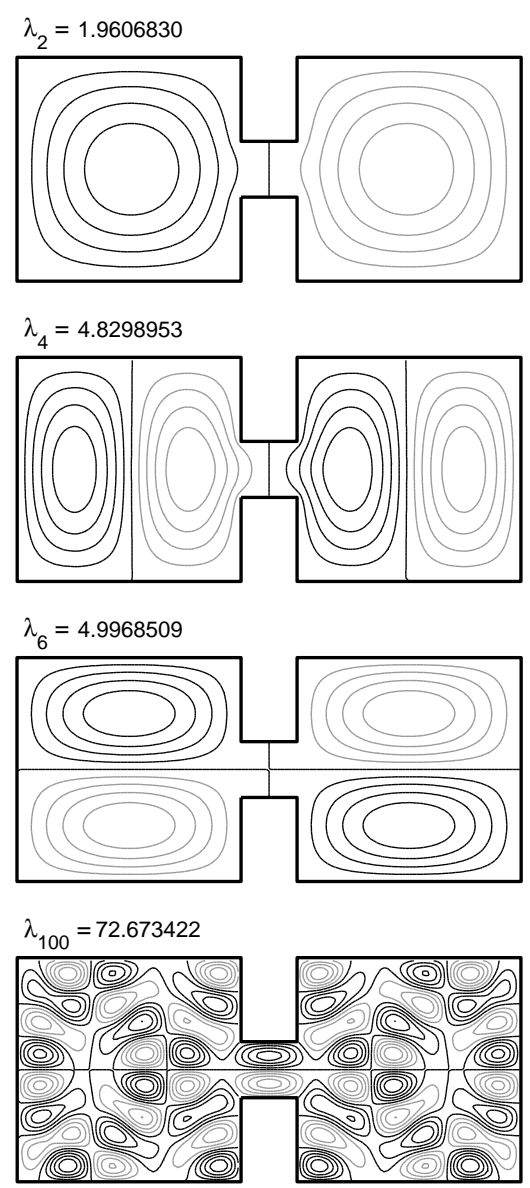

FiguRE 3. Eight eigenmodes of a rectangular dumbbell. Without the coupling between the two halves, the first six eigenvalues would be $2,2,5,5,5,5$. The coupling lowers the eigenvalues and breaks the degeneracy.

$\lambda$ increases, the eigenmodes fully penetrate the bridge region as well as the two squares.

This example can be interpreted as an illustration of the physical phenomenon of spectral line splitting. The simplest model of an atomic system might be isotropic in space, leading to eigenvalues of the Schrödinger operator appearing in degenerate pairs or triplets. This would be analogous to one or both halves of the dumbbell region, without the coupling. Higher-order effects, however, may break the symmetry and cause splitting of the lines. One of the first examples of this phenomenon was the Zeeman effect, in which the application of an external magnetic field causes spectroscopic lines to split; this discovery won the second Nobel Prize awarded in Physics, in 1902, for Lorentz and Zeeman. Twenty years later, the observation of fine structure splitting in the spectral lines of the hydrogen atom was one of the pieces of evidence that led Goudsmit and Uhlenbeck to the idea of electron spin; 

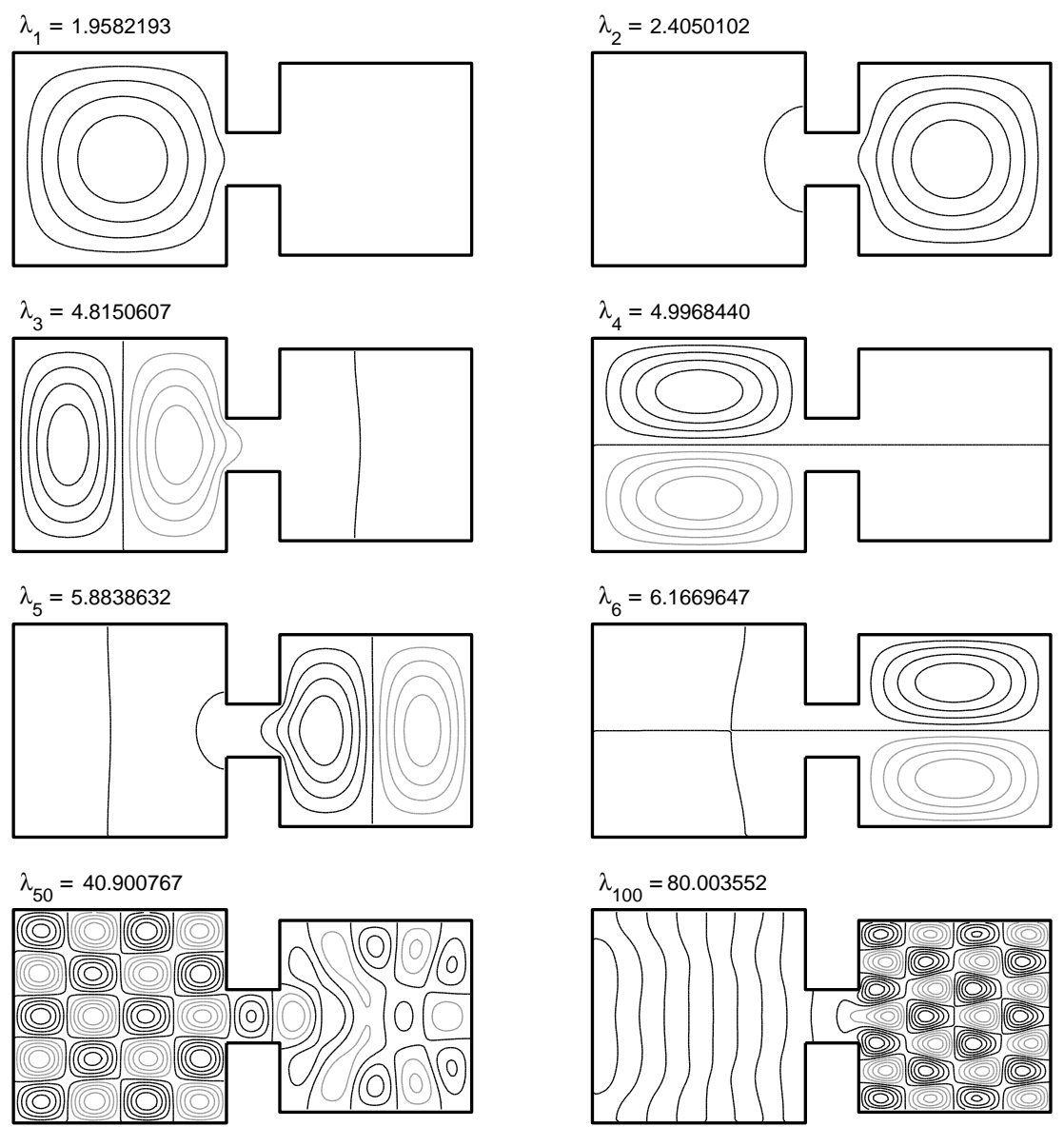

FiguRE 4. Eight eigenmodes of an asymmetrical dumbbell. The spatial variation in the drum causes localization of the lower eigenfunctions on one side or the other.

the lack of a Prize for this discovery is often cited as one of the puzzling omissions in Nobel history.

\section{An asymmetric dumbbell-localization of eigenfunctions}

Our third example is a small perturbation of the last one, but the perturbation changes the eigenvectors greatly. Here again we consider a rectangular dumbbell, but now, the square on the right has side lengths reduced from $\pi$ to $0.9 \pi$. The effects of this perturbation illustrate the general principle that whereas eigenvalues of selfadjoint problems are well-conditioned with respect to perturbations, the condition number of an eigenfunction is inversely proportional to the distance to the next nearest eigenfunction [29]. When two eigenvalues are nearly equal, the associated eigenfunctions may change wildly in response to perturbations, and in the limit of degenerate eigenvalues, they may change discontinuously (although the space they mutually span will not). 
Specifically, the difference between Figs. 3 and 4 is an elementary instance of localization: by introducing spatial variation in our system, we have caused some of the eigenmodes to be locally "pinned." Barry Simon puts it like this $[46]$ :

Thus $W$ [a perturbation] is a small flea on the elephant $V$ [the symmetrical problem]. The flea does not change the shape of the elephant. . . but it can irritate the elephant enough so that it shifts its weight, i.e.,... the ground state, instead of being asymptotically in both wells, may reside asymptotically in only one well.

Simon and others including Helffer and Sjöstrand have analyzed such effects in depth.

More remarkable examples of eigenmode localization occur in extended or infinite domains with random geometries. For example, if the dumbbell were extended to an infinite necklace of square beads of randomly varying sizes, we would (with probability 1) find eigenmodes exponentially localized. Thus the difference between Figs. 3 and 4 is a first step in the direction of the phenomenon of Anderson localization [1]. In its simplest form this effect is exhibited by a tridiagonal symmetric matrix with nonzero off-diagonal entries and random entries on the diagonal, whose eigenvectors are localized, in contrast to the global eigenvectors one encounters for a tridiagonal symmetric Toeplitz matrix. Such localization effects have fundamental importance for matters related to electrical conductivity and transparency to light in condensed matter systems, and there is another Nobel Prize in Physics in this story, awarded in 1977 to Anderson, Mott and van Vleck.

For problems of this kind there is always the balance to be considered between the strength of the coupling between entries (the width of the bridge in Figs. 3 and 4) and the strength of the spatial variation (the degree of asymmetry of the dumbbell). When the coupling is dominant the eigenvectors tend to be global, and when the variation is dominant they tend to be local. For a fixed region as in Fig. 4, though lower modes may be localized, almost all higher modes will usually be global. Indeed, for domains whose classical billiard trajectories are chaotic, Shnirelman's theorem, also known as the quantum ergodicity theorem, asserts that almost all higher modes will be global and uniformly distributed (ergodic) [44]. (The region of Fig. 4 and the other polygonal regions of the article are not in this class; because of the straight sides their classical billiard dynamics is "pseudointegrable" rather than chaotic.)

\section{An unbounded region-continuous spectrum, resonance, tunneling}

We now turn to an example of an unbounded region with infinite area. In such cases there will often be a continuous spectrum, and there may or may not be eigenfunctions in addition that correspond to bound states. Our region, shown in Fig. 5, is a semi-infinite strip of width 1 into which a slit of length $1 / 2$ has been cut at a distance 1 unit from the end.

For a semiinfinite strip without the slit, for any integer $k$ and real number $\alpha$, the function

$$
u(x, y)=\sin (k \pi y) \sin (\alpha x)
$$

satisfies the eigenvalue equation with

$$
\lambda=\pi^{2}+\alpha^{2} .
$$



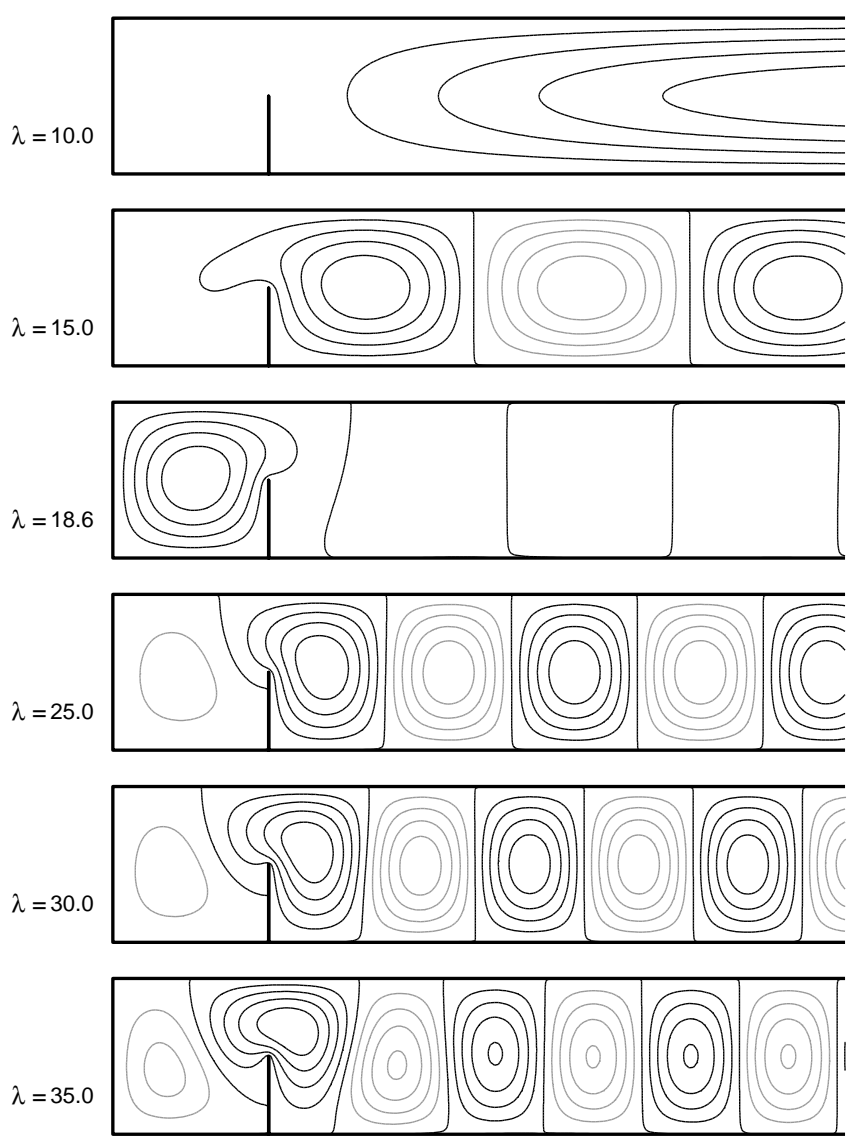

FIgURE 5. A semi-infinite slit with a resonant square cavity at the end. Each number $\lambda \geq \pi^{2}$ is in the spectrum, but the associated eigenfunctions of the continuous spectrum only penetrate significantly into the cavity when $\lambda$ is close to an eigenvalue of the square. For such values of $\lambda$, the resonance may be strong enough that the amplitude is greater in the resonant cavity than outside. Here we see this effect for $\lambda=18.6 \approx 2 \pi^{2}$ : the amplitude in the cavity is about 5.3 times as great as that outside.

This function is not square-integrable, so technically speaking, $u$ is not an eigenfunction and $\lambda$ is not an eigenvalue. However, it is common to bend terminology and call $u$ an eigenfunction anyway, or an eigenfunction of the continuous spectrum. In any case, by mollifying $u$ suitably we can make it a function in $L^{2}$ that comes arbitrarily close to satisfying the eigenvalue equation, and thus each number

$$
\lambda \in\left[\pi^{2}, \infty\right)
$$

is in the spectrum for the semiinfinite strip.

For the slit strip, the spectrum is the same, and for each $\lambda \in\left(\pi^{2}, \infty\right)$, there is again an eigenfunction of the continuous spectrum. Near the slit, these eigenfunctions have interesting behavior, as is evident in Fig. 5. For most values of $\lambda$, 


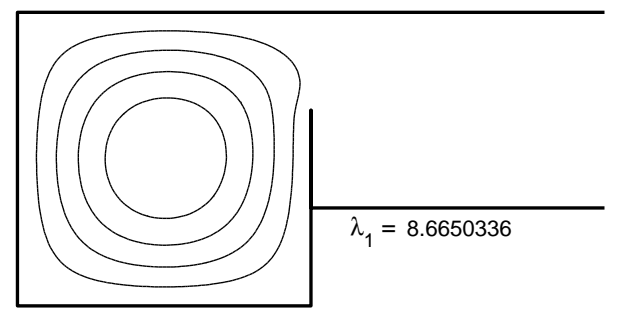

FIGURE 6. If the cavity in Figure 5 is enlarged, the region supports a bound state.

the amplitude of the eigenfunction in the cavity region is less than in the rest of $\Omega$, but for certain values, the oscillation excited in the cavity becomes as large as that outside or larger. This is the phenomenon of resonance. If the cavity were an isolated system, it would have true eigenmodes with frequencies equal to the multiples $2,5,5,8, \ldots$ of $\pi^{2}$. In fact it is weakly coupled to the infinite strip, a system that may oscillate at arbitrary frequencies above $\pi^{2}$. One might expect that there should be true eigenmodes of this operator localized in the cavity, but this cannot be so in this case. There are "quasimodes" of this form, which behave like eigenmodes for a time, but they cannot be true eigenmodes since eventually the energy in them must leak out to the rest of the system by tunneling through the gap defined by the slit. ${ }^{1}$

The problem of how to analyze situations like this, where there is an eigenmode that "ought to be there" but mathematically is not, has led to the theory of resonance in mathematical physics [34]. Physically it is of great importance, the basis of all kinds of phenomena of metastability. One of the mathematical foundations of such analysis is the WKBJ method invented in 1926. A celebrated application was Gamow's 1928 analysis of tunneling effects in quantum systems to explain the half-lives of unstable atomic nuclei. Another example is phosphorescence, a process that relies on atoms becoming excited to metastable states, not modes but quasimodes, which release their energy only slowly. Long before the quantum theory had come along to show that these effects involved modes and quasimodes, Henri Becquerel won the 1903 Nobel Prize in Physics for work related to radioactivity and phosphorescence.

\section{Unbounded regions with bound states}

Some eigenvalue problems in unbounded domains, on the other hand, possess one or more bound states that are genuine eigenfunctions, usually decaying exponentially away from their central region. In the Schrödinger equation of quantum mechanics, this is a familiar effect if a potential has a local minimum. Indeed, the quantum states of atoms and molecules are of precisely this nature.

\footnotetext{
${ }^{1}$ The term quasimode is standard in contexts like this of Hermitian operators whose eigenvectors may have surprising behavior because of degenerate or near-degenerate eigenvalues [2, 18]. Some of the fundamental results of spectral asymptotics and quantum chaos are derived by constructing quasimodes by methods of semiclassical mechanics. The alternative term pseudomode is generally used for strongly non-Hermitian operators, where modal surprises may appear even though the eigenvalues are well separated [49].
} 


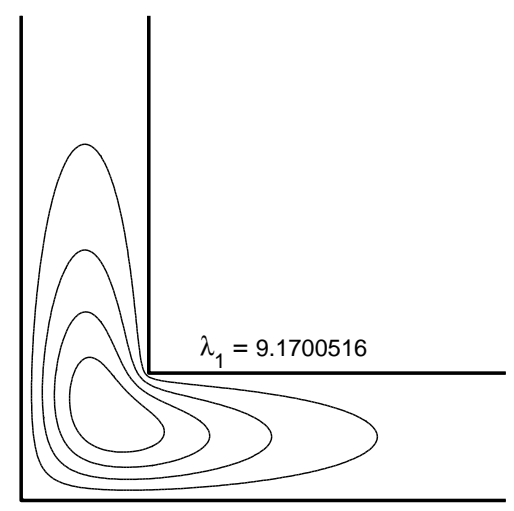

Figure 7. Bound state in an infinite "L".

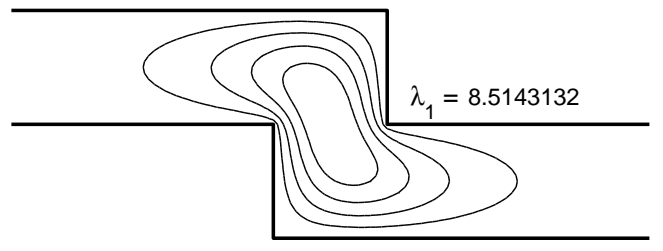

FiguRE 8. Bound state in an infinite zig-zag region.

For our problem (1.1), the analogous configuration is that of an unbounded region $\Omega$ whose portions extending to infinity have bounded width, and with a bulge or a bend in one or more positions. For example, suppose we consider the semi-infinite slit with a resonant cavity of Figure 5, but now, we increase the side length of the cavity from 1 to 1.5. The resulting region has a single bound state with eigenvalue about 8.67, shown in Figure 6. An explanation of why such states may exist is as follows. Since the cavity has size 1.5 , we can expect it approximately to admit oscillations of frequency $\lambda=2 \pi^{2} /(1.5)^{2} \approx 8.77$. This number lies below the minimal value of $\pi^{2} \approx 9.87$ for the continuous spectrum in the semiinfinite portion of the domain. Physically, a vibration at this frequency cannot tunnel out of the square cavity region; the width of the semiinfinite strip is such as to admit exponentially decaying solutions with $\lambda \approx 8.67$ but no sinusoidal solutions. In the language of the Heisenberg uncertainty principle, it could be said that a particle located in the semiinfinite part of $\Omega$, with $y$ position accordingly known to lie within 0 and 1 , could not have momentum as low as that associated with $\lambda \approx 8.77$.

Bound states such as these have been of growing interest among physicists in recent years, because nanotechnology has advanced to the point where it is possible to build quantum "corrals," "wires," "tubes" and "waveguides" that contain electrons in a quasi-two-dimensional fashion so that their quantum states are well approximated by (1.1). Celebrated images of localized states in microscopic systems have been produced by Eigler and his colleagues [21]. The book [41] is devoted to exploring another class of such problems and examines a number of interesting illustrative regions. For example, suppose we consider an "infinite L-shaped region" in the form of an infinite strip of width 1 with a sharp right-angle bend in it. As first 


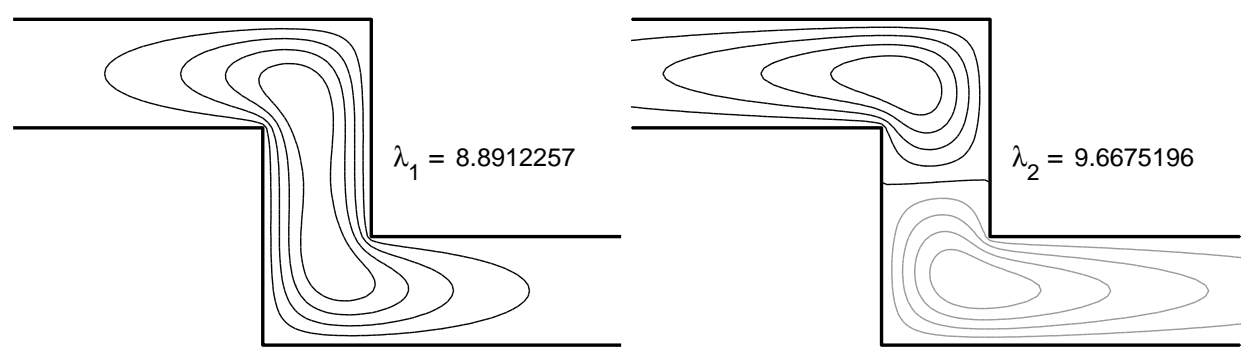

Figure 9. When the two infinite legs are separated by one more unit, there are two bound states.

shown in [39] and [45], this region has a single bound state with $\lambda \approx 9.1700516$, illustrated in Figure 7 . For the two-bend region of Figure 8 there is a bound state with $\lambda \approx 8.5143132$, and when the same region is extended by a unit square spacer in the middle, there are two bound states, as shown in Figure 9 [17]. In [41], these and other mathematical predictions are confirmed by physical experiments carried out in microwave cavities.

It has been proved that any two-dimensional "tube" of constant width, if it approaches straight channels at infinity and contains a bend at a finite location, must sustain at least one bound state $[\mathbf{2 4}, \mathbf{2 8}]$.

\section{Eigenvalue avoidance or level repulsion}

Except for $\lambda_{1}$, any eigenvalue of (1.1) can be degenerate. As we have discussed, degeneracies typically (but not always [12]) arise when $\Omega$ has multiple lines of symmetry. For example, it is easily seen that rectangular domains may have numerous degeneracies, and this was the explanation for the effect $\lambda_{103}=\lambda_{104}=\lambda_{105}$ in Figure 1. In rectangles the vertical and horizontal directions decouple, and it is no surprise if degenerate eigenvalues appear in systems that decouple into two or more independent subsystems.

Generically, however, the problem (1.1) tends to avoid degenerate eigenvalues, and this is a phenomenon that has been analyzed in detail since it was first noted by von Neumann and Wigner in $1929[\mathbf{3 , 4 8 , 5 2}$. The following elementary argument captures the essence of the matter. The set of real symmetric $2 \times 2$ matrices is a vector space of dimension 3 :

$$
\left\{\left(\begin{array}{ll}
a & b \\
b & c
\end{array}\right)\right\}, \quad a, b, c \in \mathbf{R} .
$$

The set of real symmetric $2 \times 2$ matrices whose eigenvalues are degenerate, however, is a subspace of dimension just one:

$$
\left\{\left(\begin{array}{ll}
a & 0 \\
0 & a
\end{array}\right)\right\}, \quad a \in \mathbf{R}
$$

The codimension of the set of degenerate matrices is thus 2 , not 1 as one might have expected. It follows that for generic $2 \times 2$ real symmetric matrix problems dependent on a single real parameter $\alpha$, one cannot expect to find a value of $\alpha$ for which there will be a degenerate pair of eigenvalues. Essentially the same argument works for $n \times n$ matrices, and the same effect occurs also with problem (1.1). Triple eigenvalues 

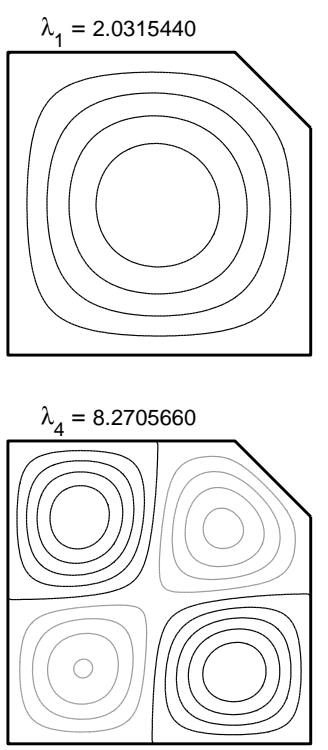
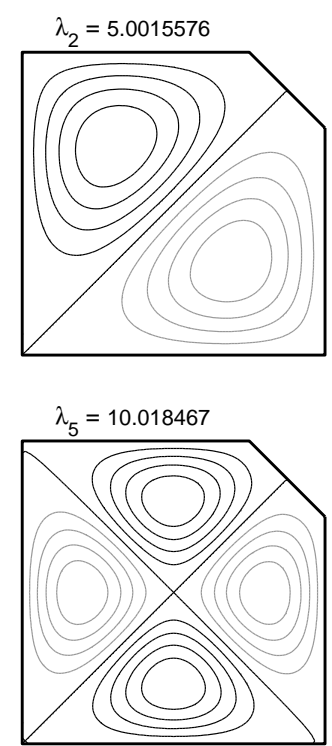
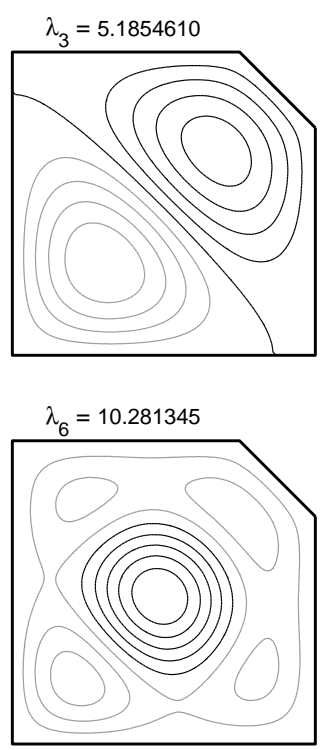

Figure 10. A square of side length $\pi$, but with the corner snipped to break the symmetry.

generically require 5 parameters, quadruple eigenvalues need 9 , quintuples need 14 , and so on.

One of the many reasons for interest in eigenvalue avoidance is that it may offer a window into the behavior of the Riemann zeta function. It is an old idea, known as the Hilbert-Pólya conjecture, that the zeros of the zeta function on the critical line $\operatorname{Re} z=\frac{1}{2}$ might be the eigenvalues of some operator. Extensive numerical experiments have shown that these zeros appear to be distributed in a fashion close to that of the eigenvalues of random Hermitian matrices $[42,43]$. (In the complex Hermitian case the avoidance effect is even stronger, as the codimension increases from 2 to 3.) This discovery has generated a great amount of research activity, which one day, perhaps, might lead to a proof of the Riemann hypothesis $[\mathbf{1 1}, \mathbf{3 6}, \mathbf{3 7}$. Closer to the topic of this article, it has been conjectured that if the region $\Omega$ is one whose classical billiard trajectories are chaotic, then its eigenvalues match statistics for random real symmetric matrices $[\mathbf{1 6}]$.

Figure 10 gives an illustration of the eigenvalue avoidance effect. ${ }^{2}$ For a square of side length $\pi$, the eigenvalues will be $2,5,5,8,10,10, \ldots$, with multiple degeneracies attributable to the symmetry. Suppose now that we break the symmetry by snipping the upper-right corner of the square, inserting a new boundary segment between points a distance $\pi / 4$ from the corner along the upper and right sides. In the figure we see that the degeneracy of $\lambda_{3}$ and $\lambda_{4}$ has been broken, and these eigenvalues now differ by about $3.7 \%$. This effect is much like what we saw already in Figure. 3.

Figure 11 shows the influence of the snipped corner on the dependence of the eigenvalues on a parameter. On the left, the first nine eigenvalues are plotted for

\footnotetext{
${ }^{2}$ The idea of considering problems like this was suggested to us by Rainer Hempel of the Technical University of Braunschweig. For another computed example see [14].
} 

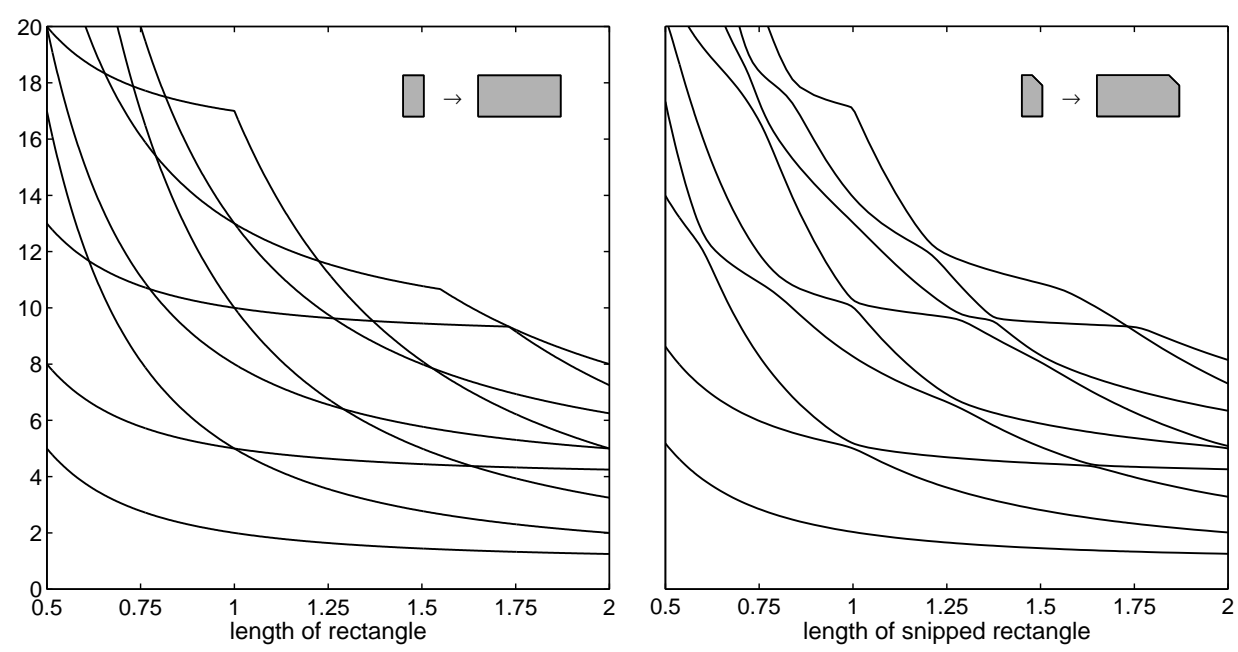

FiguRE 11. The first nine eigenvalues for rectangles and snipped rectangles of height 1 and varying length. For the snipped rectangles, though this is not clear from the figure in all cases, the curves have no intersections.

rectangles of height $\pi$ and length ranging from $\pi / 2$ to $2 \pi$. As the length increases, all the eigenvalues decrease monotonically, but the rates are quite uneven and there are a number of degeneracies. On the right, the analogous plot is presented for rectangles with a corner of side length $\pi / 4$ snipped away. The curves lie roughly in the same positions, but their topology has changed entirely: the intersections are gone.

\section{Isospectral drums}

One of the celebrated examples of problem (1.1) stems from Mark Kac's Chauvenet Prize-winning 1966 article, "Can one hear the shape of a drum?" [35]. Kac was asking a uniqueness question, whether there could be two distinct regions $\Omega_{1}$ and $\Omega_{2}$, not related by rotation or reflection, with all identical eigenvalues. In 1992 Gordon, Webb and Wolpert shown that the answer is no, and Figure 12 shows one of their examples [27]. The shape on the left and that one the right are different, but all their eigenvalues are identical. The eigenvalues and eigenfunctions for these regions were first computed by Driscoll [23].

\section{Maximizing $\lambda_{3} / \lambda_{1}$}

Finally, we consider a problem of eigenvalue optimization. As is well known, there are all kinds of constraints on the spacing and separation of the eigenvalues of a system (1.1). A specific question one may ask is, how large can the ratio $\lambda_{2} / \lambda_{1}$ be? The longstanding Payne-Pólya-Weinberger conjecture was this the maximum was achieved by a disk, for which we have

$$
\frac{\lambda_{2}}{\lambda_{1}}=\frac{j_{1,1}^{2}}{j_{0,1}^{2}} \approx 2.539,
$$



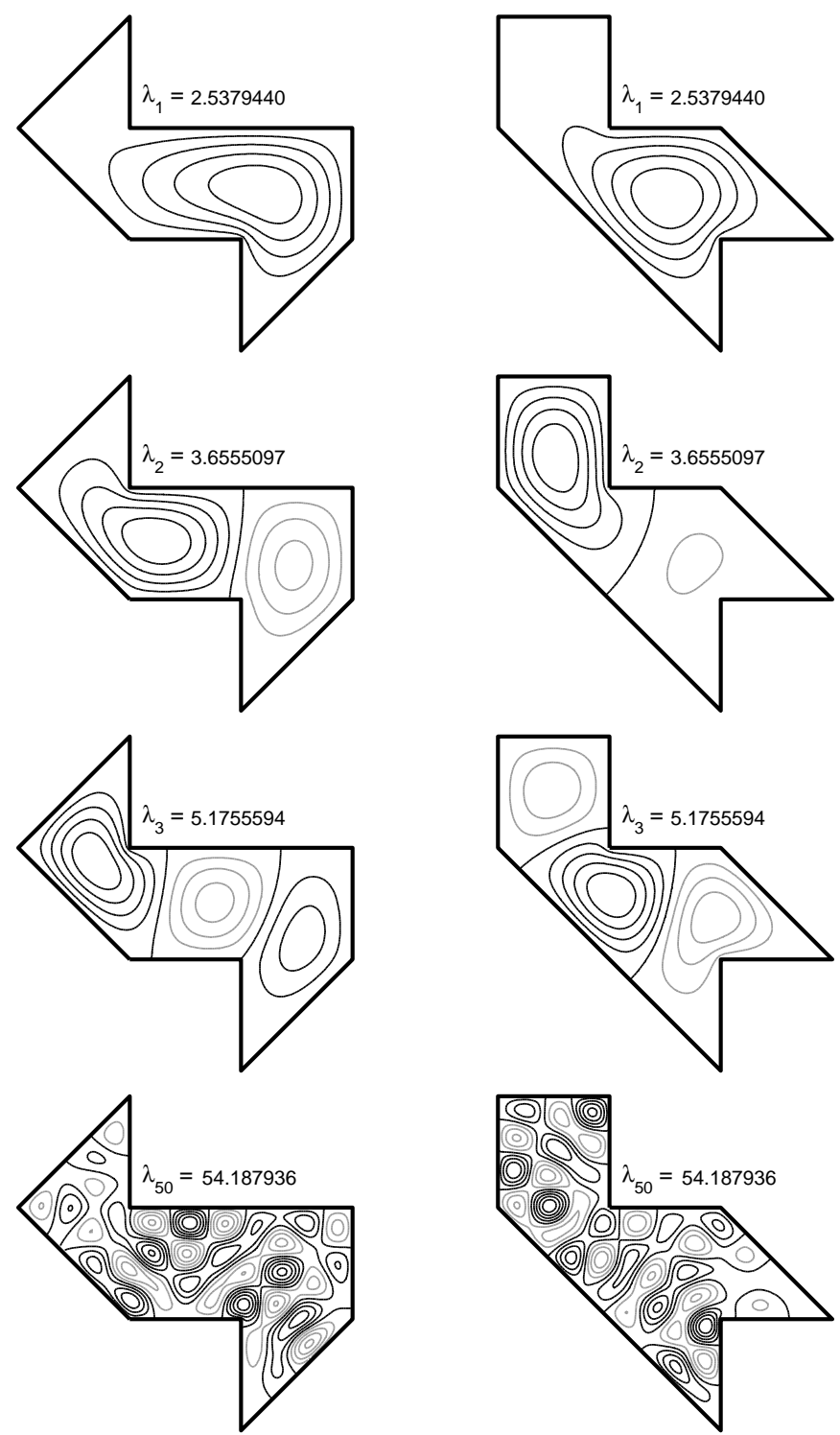

Figure 12. Isospectral drums of Gordon, Webb and Wolpert [27]. Eigenmodes like these were observed experimentally by Sridhar and Kudrolli $[\mathbf{4 7}]$ and computed by Driscoll $[\mathbf{2 3}]$.

where $j_{k, 1}$ represents the first zero of the Bessel function $J_{k}$. This conjecture was turned into a theorem by by Ashbaugh and Benguria [4].

What about $\lambda_{3} / \lambda_{1}$ ? Maximizing this ratio is an open problem; the maximum is certainly not achieved by a disk. The best estimate up to now appears to be due to Levitin and Yagudin [40], who propose a certain dumbbell shape for which the ratio appears to be about 3.202. Figure 13 shows another dumbbell shape whose ratio $\lambda_{3} / \lambda_{1}$ falls short of this value by about $0.35 \%$ and is thus apparently not quite 


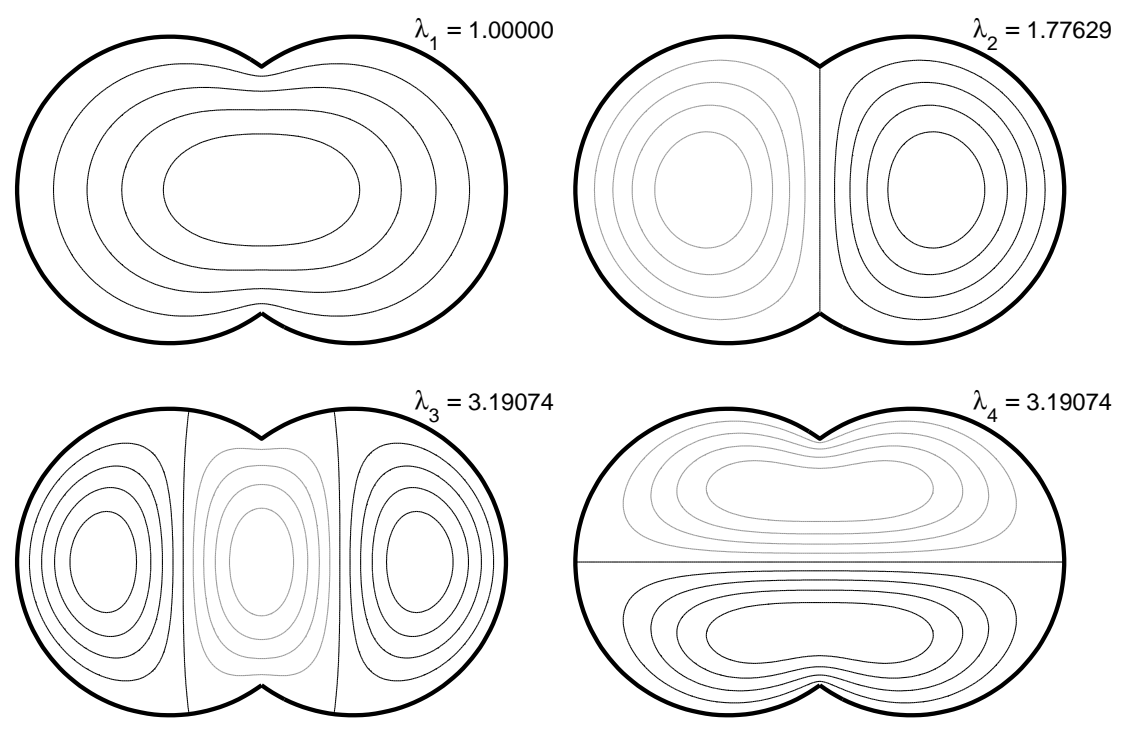

FIgURE 13. A two-disk domain with a high ratio $\lambda_{3} / \lambda_{1}$.

optimal. It is, however, very simple, consisting of the union of the disks of radius 2.002324 centered at $x= \pm 1.191321$. To six-digit accuracy at least, this region has a degenerate eigenvalue pair $\lambda_{3}=\lambda_{4}$.

\section{Acknowledgments}

We are grateful for advice from a number of people in preparing this article, including Alexander Barnett, Raphael Benguria, Brian Davies, Toby Driscoll, Pavel Exner, Jonathan Keating, Michael Levitin, Tim Londergan, and Barry Simon.

\section{References}

[1] P. W. Anderson, Absence of diffusion in certain random lattices, Phys. Rev. 109 (1958), 1492-1505.

[2] V. I. Arnold, Modes and quasi-modes, Func. Anal. Applics. 6 (1972), 94-101.

[3] V. I. Arnold, Mathematical Methods of Classical Mechanics, Springer, New York, 1978.

[4] M. S. Ashbaugh and R. D. Benguria, Proof of the Payne-Pólya-Weinberger conjecture, Bull. Amer. Math. Soc. 25 (1991), 19-29.

[5] A. Bäcker, Numerical aspects of eigenvalue and eigenfunction computations for chaotic quantum systems, in The Mathematical Aspects of Quantum Maps, M. Degli Esposti and S. Graffi, eds., Lect. Notes Phys. 618, Springer, Berlin, 2003, pp. 91-144.

[6] H. P. Baltes and E. R. Hilf, Spectra of Finite Systems, Bibliographisches Inst. Wissenschaftsverlag, Mannheim, 1976.

[7] L. Banjai, Eigenfrequencies of fractal drums, Prepr. 11, Max-Planck-Inst. Math. Naturwiss., Leipzig, 2005.

[8] A. H. Barnett, Dissipation in Deforming Chaotic Billiards, PhD thesis, Dept. of Physics, Harvard University, 2000.

[9] A. H. Barnett, Improved inclusion bounds for high frequency Dirichlet eigenvalues with a generalized Method of Particular Solutions, manuscript, 2005.

[10] M. V. Berry and C. J. Howls, High orders of the Weyl expansion for quantum billiards: resurgence of periodic orbits, and the Stokes phenomenon, Proc. Roy. Soc. A 447 (1994), $527-555$. 
[11] M. V. Berry and J. P. Keating, The Riemann zeros and eigenvalue asymptotics, SIAM Rev. 41 (1999), 236-266.

[12] M. V. Berry \& M. Wilkinson, Diabolical points in the spectra of triangles, Proc. Roy. Soc. Lond. A 392 (1984), 15-43.

[13] T. Betcke, Numerical computation of eigenfunctions of planar regions, D. Phil. diss., Oxford U. Computing Lab., 2005.

[14] T. Betcke \& L. N. Trefethen, Computations of eigenvalue avoidance in planar domains, Proc. Appl. Math. Mech. 4 (2004), 634-635.

[15] T. Betcke \& L. N. Trefethen, Reviving the method of particular solutions, SIAM Rev. 47 (2005), 469-491.

[16] O. Bohigas, M. J. Gianoni, and C. Schmit, Characterization of chaotic quantum spectra and universality of level fluctuation laws, Phys. Rev. Lett. 52 (1984), 1-4.

[17] J. P. Carini, J. T. Londergan, D. P. Murdock, D. Trinkle and C. S. Yung, Bound states in waveguides and bent quantum wires. I. Applications to waveguide systems, Phys. Rev. B 55 (1997), 9842-9851.

[18] Y. Colin de Verdière, Quasi-modes sur les variétés Riemanniennes, Invent. Math. 43 (1977), $15-22$.

[19] D. L. Colton, Analytic Theory of Partial Differential Equations, Pitman, Boston, 1980

[20] R. Courant and D. Hilbert, Methods of Mathematical Physics, v. 1, Interscience, 1953.

[21] M. F. Crommie, C. P. Lutz and D. M. Eigler, Confinement of electrons to quantum corrals on a metal surface, Science 262 (1993), 218-221.

[22] J. Descloux and M. Tolley, An accurate algorithm for computing the eigenvalues of a polygonal membrane, Comp. Meth. Appl. Mech. Engr. 39 (1983), 37-53.

[23] T. A. Driscoll, Eigenmodes of isospectral drums, SIAM Rev. 39 (1997), 1-17.

[24] P. Exner and P. Seba, Bound states in curved quantum waveguides, J. Math. Phys. 30 (1989), 2574-2580.

[25] L. Fox, P. Henrici and C. Moler, Approximations and bounds for eigenvalues of elliptic operators, SIAM J. Numer. Anal. 4 (1967), 89-102.

[26] P. Garabedian, Applications of analytic continuation to the solution of boundary value problems, J. Ratl. Mech. Anal. 3 (1954), 383-393.

[27] C. Gordon, D. Webb and S. Wolpert, Isospectral plane domains and surfaces via Riemannian orbifolds, Invent. Math. 110 (1992), 1-22.

[28] J. Goldstone and R. L. Jaffe, Bound states in twisting tubes, Phys. Rev. B 45 (1992), 1410014107.

[29] G. H. Golub and C. F. Van Loan, Matrix Computations, 3rd ed., Johns Hopkins U. Press, Baltimore, 1996

[30] M. Gutzwiller, Quantum chaos, Scientific American, Jan. 1992.

[31] M. C. Gutzwiller, Chaos in Classical and Quantum Mechanics, Springer, New York, 1990.

[32] E. J. Heller, in Wavepacket Dynamics and Quantum Chaology, M. J. Giannoni, A. Voros and J. Zinn-Justin, eds., Proc. 1989 Les Houches Summer School on Chaos and Quantum Physics, Elsevier, 1991, pp. 547-663.

[33] P. Henrici, A survey of I. N. Vekua's theory of elliptic partial differential equations with analytic coefficients, Z. Ang. Math. Phys. 8 (1957), 169-203.

[34] P. D. Hislop and I. M. Sigal, Introduction to Spectral Theory, With Applications to Schrödinger Operators, Springer, New York, 1996.

[35] M. Kac, Can one hear the shape of a drum?, Amer. Math. Monthly 73 part II (1966), 1-23.

[36] N. M. Katz and P. Sarnak, Random Matrices, Frobenius Eigenvalues, and Monodromy, Amer. Math. Soc., Providence, RI, 1999.

[37] J. P. Keating and N. C. Snaith, Random matrix theory and $\zeta(1 / 2+i t)$, Comm. Math. Phys. 214 (2000), 57-89.

[38] J. R. Kuttler and V. G. Sigillito, Eigenvalues of the Laplacian in two dimensions, SIAM Rev. 26 (1984), 163-193.

[39] F. Lenz, et al., Quark confinement and hadronic interactions, Ann. Phys. 170 (1986), 65-254.

[40] M. Levitin and R. Yagudin, Range of the first three eigenvalues of the planar Dirichlet Laplacian, LMS J. Comput. Math. 6 (2003), 1-17.

[41] J. T. Londergan, J. P. Carini and D. P. Murdock, Binding and Scattering in Two-Dimensional Systems, Springer, Berlin, 1999. 
[42] H. L. Montgomery, The pair correlation of zeros of the zeta function, in H. G. Diamond., ed., Analytic Number Theory, Proc. Symp. Pure Math. 24, Amer. Math. Soc., Providence, RI, 1977, pp. 181-193.

[43] A. M. Odlyzko, The $10^{22}{ }^{-}$nd zero of the Riemann zeta function, in M. van Frankenhuysen and M. L. Lapidus, eds., Dynamical, Spectral, and Arithmetic Zeta Functions, Amer. Math. Soc., Contemp. Math. 290 (2001), pp. 139-144.

[44] A. I. Shnirelman, Ergodic properties of eigenfunctions, Usp. Mat. Nauk. 29 (1974), 181-182.

[45] R. L. Schult, D. G. Ravenhall and H. W. Wyld, Quantum bound states in a classically unbound system of crosed wires, Phys. Rev. B 39 (1989), 5476-5479.

[46] B. Simon, Semiclassical analysis of low loying eigenvalues. IV. The flea on the elephant, J. Funct. Anal. 63 (1985), 123-136.

[47] S. Sridhar and A. Kudrolli, Experiments on not "hearing the shape" of drums, Phys. Rev. Lett. 72 (1994), 2175-2178.

[48] M. Teytel, How rare are multiple eigenvalues?, Comm. Pure Appl. Math. 52 (1999), 917-394.

[49] L. N. Trefethen and M. Embree, Spectra and Pseudospectra: The Behavior of Nonnormal Matrices and Operators, Princeton U. Press, Princeton, 2005.

[50] I. N. Vekua, New Methods for Solving Elliptic Equations, North-Holland, Amsterdam, 1967.

[51] E. Vergini and M. Saraceno, Calculation by scaling of highly excited states of billiards, Phys. Rev. E 52 (1995), 2204-2207.

[52] J. von Neumann and E. Wigner, Über das Verhalten von Eigenwerten bei Adiabatischen Prozessen, Z. Phys. A 30 (1929), 467-470.

Oxford Computing Laboratory, Wolfson Bldg., Parks Rd., Oxford OX1 3QD, UK E-mail address: LNT@comlab.ox.ac.uk

Institut Computational Mathematics, tU Braunschweig, Pockelsstr. 14, D-38023 Braunschweig, Germany

E-mail address: t.betcke@tu-bs.de 